\title{
Target Identification and Mode of Action of Four Chemically Divergent Drugs against Ebolavirus Infection
}

\author{
Jingshan Ren, ${ }^{\dagger, \#}$ Yuguang Zhao, ${ }^{\dagger, \#}$ Elizabeth E. Fry, ${ }^{\dagger}$ and David I. Stuart ${ }^{*}, \dagger,{ }^{+}$
}

${ }^{\dagger}$ Division of Structural Biology, University of Oxford, The Henry Wellcome Building for Genomic Medicine, Headington, Oxford, OX3 7BN, U.K.

${ }^{\ddagger}$ Diamond Light Source Ltd., Harwell Science \& Innovation Campus, Didcot, OX11 0DE, U.K.

Supporting Information
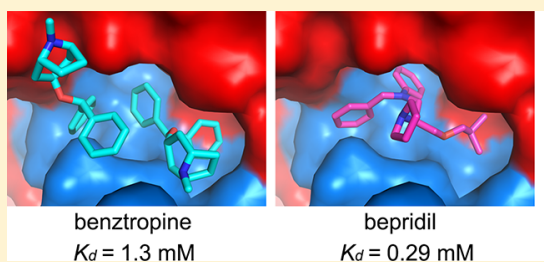

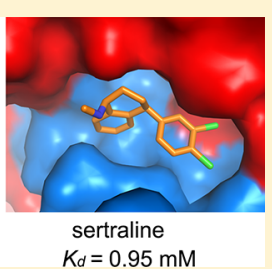

ABSTRACT: Here, we show that four chemically divergent approved drugs reported to inhibit Ebolavirus infection, benztropine, bepridil, paroxetine and sertraline, directly interact with the Ebolavirus glycoprotein. Binding of these drugs destabilizes the protein, suggesting that this may be the mechanism of inhibition, as reported for the anticancer drug toremifene and the painkiller ibuprofen, which bind in the same large cavity on the glycoprotein. Crystal structures show that the position of binding and the mode of interaction within the pocket vary significantly between these compounds. The binding constants $\left(K_{\mathrm{d}}\right)$ determined by thermal shift assay correlate with the protein-inhibitor interactions as well as with the antiviral activities determined by virus cell entry assays, supporting the hypothesis that these drugs inhibit viral entry by binding the glycoprotein and destabilizing the prefusion conformation. Details of the protein-inhibitor interactions of these complexes and their relation with binding affinity may facilitate the design of more potent inhibitors.

\section{INTRODUCTION}

Ebolavirus (EBOV) and Marburgvirus (MARV) belong to the family Filoviridae and cause severe, often fatal, diseases. There are currently no vaccines or drugs available to combat the diseases. The family Filoviridae comprises currently seven species in three genera (Cuevavirus, Ebolavirus, and Marburgvirus). ${ }^{1}$ Most of these viruses infect humans to cause severe hemorrhagic fever with a high fatality rate. ${ }^{2}$ The cost of the 2013-2016 outbreak of Ebolavirus in West Africa, to both human life and the economy, was unprecedented. ${ }^{3}$ Filoviruses are single-stranded negative-sense RNA viruses with a membrane envelope decorated by trimers of a glycoprotein (GP, cleaved by furin to form GP1 and GP2 subunits), which is solely responsible for host cell attachment, endosome entry, and membrane fusion. ${ }^{4-8}$ GP1 is responsible for binding to the receptor NPC1, and GP2 is a class I viral fusion protein catalyzing fusion of the viral and host membrane. The structures of Ebolavirus GP and its complexes with Fabs and receptor NPC1 have been previously described. ${ }^{9-11}$ In the prefusion state three copies of GP (each composed of GP1 and GP2 subunits) form the biological trimer with the receptorbinding site of GP1 being protected by a glycan cap (Figure 1). In the late endosome/lysosome the glycan cap is removed by cathepsin $\mathrm{B} / \mathrm{L}$ to allow binding of the receptor, which subsequently triggers the uncoupling of GP2 from GP1 and leads to membrane fusion driven by large conformational changes, which ultimately lead to the radically different postfusion state. ${ }^{10,12}$ In the apo prefusion structure of EBOV GP, each subunit harbors a tunnel, and the three tunnels of the trimer join at the three-fold axis. The tunnel entrance is capped by a tight turn called the DFF lid. ${ }^{13}$

A surprisingly large number of Food and Drug Administration (FDA) approved drugs have been found to be active against Ebolavirus infection using either infectious Ebolavirus replication or pseudovirus entry assays. ${ }^{14-20}$ These drugs have various primary pharmacological targets and huge chemical diversity, and the mechanism of EBOV inhibition is largely unknown. Furthermore, given their weak inhibitory properties, these drugs may not be adequate to curtail EBOV infection. We have shown in previous studies that the anticancer drug toremifene and the painkiller ibuprofen both bind at the entrance of the tunnel of the EBOV GP pushing aside the DFF lid, and that inhibitor binding decreases the thermal stability of the GP. ${ }^{13}$ Here, we show, by crystallography, that another four FDA approved drugs that inhibit EBOV infection directly bind in the same cavity on the EBOV GP as toremifene. We also show, using thermal shift assay, that they decrease the thermal stability of the protein. Each of the drugs, due to their divergent chemical structures, exploits a different portion of the spatial volume of the cavity and makes distinct protein interactions. Structural analysis of these GP-drug complexes underlines key

Received: August 22, 2017

Published: December 22, 2017 

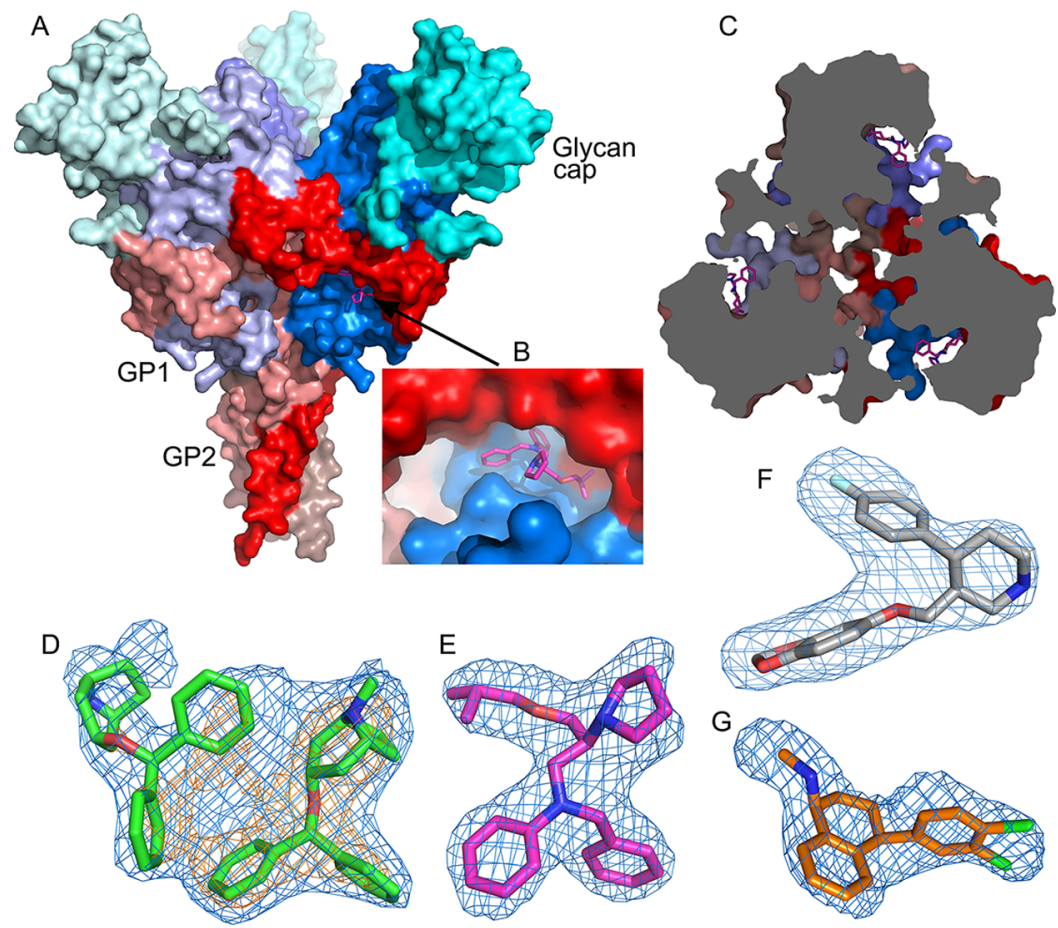

Figure 1. Overall structure of EBOV GP and electron density maps. (A) Surface representation of the EBOV GP trimer; GP1 is in blue, GP2 in red, and the glycan cap in cyan. The bound bepridil at the entrance of a tunnel is shown as magenta sticks. (B) Close-up of the tunnel entrance with bound bepridil. (C) Slice of EBOV GP trimer perpendicular to the three-fold of the trimer as viewed toward the viral membrane showing the three tunnels, each from a monomer, connected at the three-fold; bepridils at the channel entrances are in magenta sticks. (D-G) Simulated annealing $\mid F_{\mathrm{o}}$ $-F_{c}$ omit electron density maps for benztropine (D), bepridil (E), paroxetine (F), and sertraline $(G)$ contoured at $3.5 \sigma$; the orange density in $(D)$ is contoured at $6 \sigma$ showing that one benztropine molecule is less well ordered.

volumes of the binding cavity and protein-inhibitor interactions, which can help guide the design of more potent inhibitors.

\section{RESULTS AND DISCUSSION}

Identification of Drugs That Interact Directly with EBOV GP. Eight previously reported FDA approved drugs that inhibit EBOV entry were evaluated to determine if they directly interact with EBOV GP using thermal shift assays. The experiment was carried out at $\mathrm{pH} 5.2$, which is close to the physiological $\mathrm{pH}$ of the late endosomal where the fusion takes place and also the $\mathrm{pH}$ at which the EBOV GP crystals were grown. The results show that four of these, the anticholinergic/ antihistamine agent benztropine, ${ }^{15,16,21,22}$ antianginal bepri$\mathrm{dil}_{1}{ }^{15,16}$ and antidepressants paroxetine $\mathrm{e}^{15,23}$ and sertraline $\mathrm{e}^{15,16}$ decrease the melting temperature $\left(T_{\mathrm{m}}\right)$ of EBOV GP by up to $6,6,4$, and $3{ }^{\circ} \mathrm{C}$, respectively, at $500 \mu \mathrm{M}$ concentration (Figures 2 and 3; Figure S1 in Supporting Information). Other drugs $^{15}$ tested, aripiprazole, astemizole, efavirenz and quinacrine, showed either marginal or no effect on the thermal stability of EBOV GP, suggesting weak or no direct binding. The binding constants, $K_{d}$ s, derived from the thermal-shift assay are $1.3 \mathrm{mM}$ for benztropine, $0.29 \mathrm{mM}$ for bepridil, 0.65 $\mathrm{mM}$ for paroxetine, and $0.95 \mathrm{mM}$ for sertraline. Note that this assumes a 1:1 binding model; however, as described below, two molecules of benztropine bind to each binding pocket so the calculation may underestimate the strength of attachment (for instance, in the case of ordered binding). Compared with toremifene $\left(\Delta T_{\mathrm{m}}=15{ }^{\circ} \mathrm{C}, K_{\mathrm{d}}=16 \mu \mathrm{M}\right)$, the four inhibitors identified here in general are weaker binders and show correspondingly weaker thermal shifts and $K_{d} s$ (Table 1).<smiles>CN1C2CCC1CC(OC(c1ccccc1)c1ccccc1)C2</smiles><smiles>Fc1ccc(C2CCNCC2COc2ccc3c(c2)OCO3)cc1</smiles>

paroxetine
B<smiles>CC(C)COCC(CN(Cc1ccccc1)c1ccccc1)N1CCCC1</smiles>

bepridil

$\mathrm{D}$<smiles>CN[C@H]1CC[C@@H](c2ccc(Cl)c(Cl)c2)c2ccccc21</smiles>

sertraline

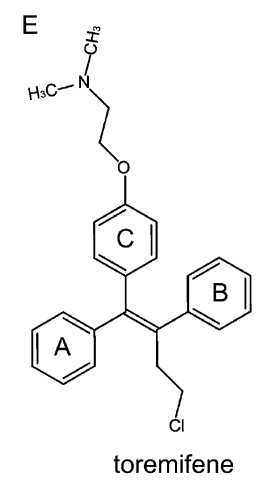

Figure 2. Chemical structures. (A) Benztropine, (3-endo)-3(diphenylmethoxy)-8-methyl-8-azabicyclo[3.2.1]octane. (B) Bepridil, 1-[2-(N-benzylanilino)-1-(isobutoxymethyl)ethyl]pyrrolidine. (C) $\mathrm{Pa}$ roxetine, $(3 S, 4 R)-3$-[(1,3-benzodioxol-5-yloxy)methyl]-4-(4fluorophenyl)piperidine. (D) Sertraline, $(1 S, 4 S)$-4-(3,4-dichlorophenyl)- $N$-methyl-1,2,3,4-tetrahydro-1-naphthalenamine. (E) Toremifene, 2-[4-[(Z)-4-chloro-1,2-diphenylbut-1-enyl]phenoxy]-N,N-dimethylethanamine.

The reported $\mathrm{IC}_{50}$ is $3 \mu \mathrm{M}$ for sertraline compared to $5 \mu \mathrm{M}$ for bepridil in Vero cells; however, bepridil provided better protection than sertraline in a mouse model. ${ }^{15}$ As negative controls we also performed the thermal shift analysis using two further inhibitors, strophanthin and U18666A. Strophanthin inhibits EBOV infection but is not an EBOV entry inhibitor, ${ }^{15}$ while U1866A inhibits viral entry by interacting with the 

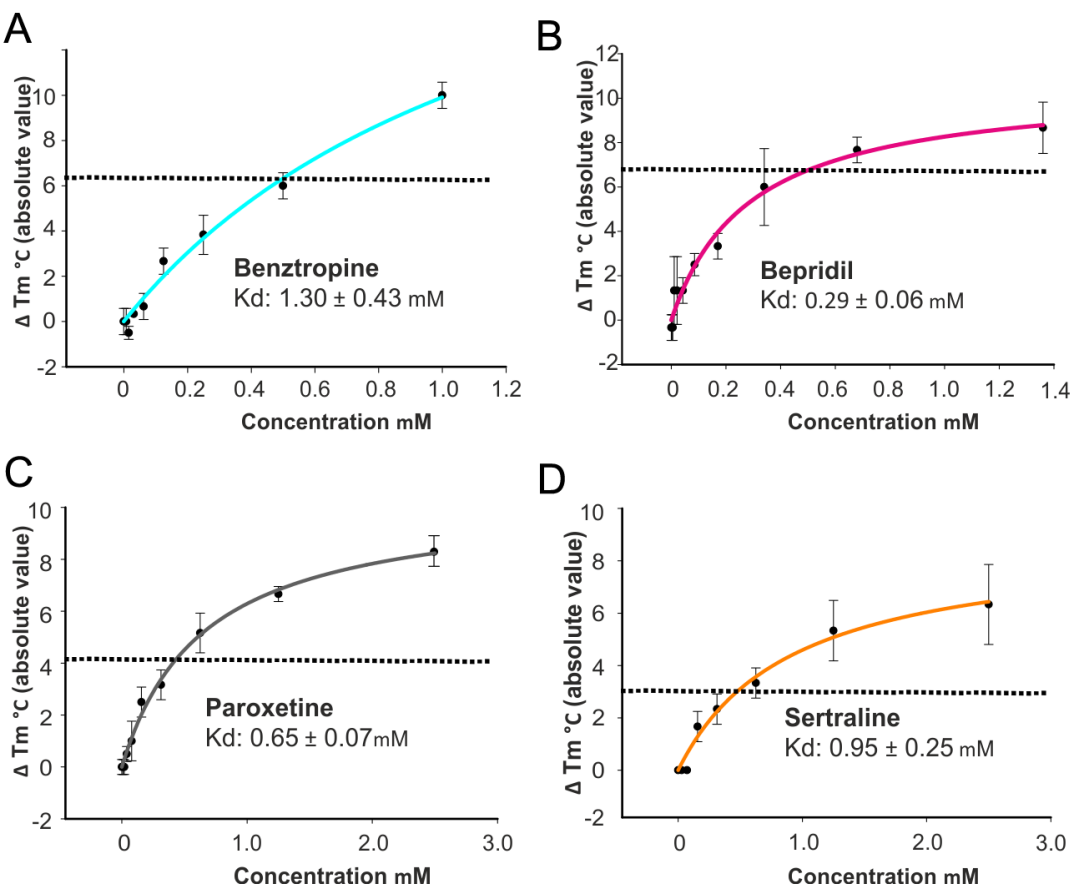

Figure 3. Summary of thermal shift assay. Shifts in melting temperature $\left(\Delta T_{\mathrm{m}}\left({ }^{\circ} \mathrm{C}\right)\right.$ in absolute value $)$ plotted against concentrations of benztropine (A), bepridil (B), paroxetine (C), and sertraline (D) at $\mathrm{pH}$ 5.2. Data are mean $\pm \mathrm{SD}(n=3)$. The dotted line in each panel indicates $\Delta T_{\mathrm{m}}$ at $0.5 \mathrm{mM}$ of the drug. The affinity constant $K_{\mathrm{d}}$ is calculated by a ligand binding 1:1 saturation, fitted with the SigmaPlot version 13 (Systat Software Inc.). The raw data are shown in Figure S2.

Table 1. Binding Affinity $\left(K_{\mathrm{d}}\right)$, Protein Stability Change $\left(\Delta T_{\mathrm{m}}\right)$, and Half-Maximum Inhibitory Concentration $\left(\mathrm{IC}_{50}\right)$

\begin{tabular}{lccll} 
& & & \multicolumn{2}{c}{$\mathrm{IC}_{50}(\mu \mathrm{M})^{b}$} \\
\cline { 4 - 5 } \multicolumn{1}{c}{ inhibitor } & $K_{\mathrm{d}}(\mu \mathrm{M})$ & $\Delta T_{\mathrm{m}}\left({ }^{\circ} \mathrm{C}\right)^{a}$ & \multicolumn{1}{c}{ Vero E6 } & \multicolumn{1}{c}{ HepG2 } \\
toremifene & 16 & -15 & $0.162(0.048)$ & $0.026(0.0013)$ \\
benztropine & 1300 & -6 & $8.07(0)$ & $2.82(0.13)$ \\
bepridil & 290 & -6 & $5.08(0.38)$ & $3.21(0.15)$ \\
paroxetine & 650 & -4 & $7.45(0.41)$ & $1.38(0.076)$ \\
sertraline & 950 & -3 & $3.13(0.24)$ & $1.44(0.057)$ \\
${ }^{a} \Delta T_{\mathrm{m}}$ is measured at inhibitor concentration of $500 \mu \mathrm{M} .{ }^{b} \mathrm{IC}_{50}$ values \\
and standard deviations (in parentheses) are adapted from Johansen et \\
al. $^{15}$
\end{tabular}

receptor $\mathrm{NPC1} .^{24}$ As expected neither strophanthin nor U1866A alter the melting temperature of EBOV GP (Figure S1).

Overall Structures of EBOV GP-Drug Complexes. The four drugs were separately prepared in crystallization liquor, and crystals of EBOV GP were soaked in these solutions before collecting X-ray diffraction data, which allowed structure determination, revealing that all four compounds bind EBOV GP (Experimental Section). X-ray data sets, all extending to 2.4 $\AA$ resolution or better and measured with high redundancy (Table S1), were collected at the Diamond synchrotron. The overall structures of the glycoprotein component of these four complexes are very similar to each other with rmsds less than $0.6 \AA$ for all $\mathrm{C}_{\alpha}$ atoms of the protein. Each bound drug has good electron density allowing its conformation to be defined (Figure 1). Significant conformational differences are observed at residues $46-52$ of GP1, and 521-525 and 577-583 of GP2 (Figure S2). Residues 46-52 precede the disulfide bond (C53-C609) between GP1 and GP2 and have two conformations in the GP-paroxetine complex, one correspond- ing to that observed in apo GP, GP-toremifene, and GPbepridil, and the other to that seen in GP-ibuprofen, GPbenztropine, and GP-sertraline. Residues 577-583 that link $\alpha 3$ and $\alpha 4$ move about $6 \AA$ by rotation around the three-fold axis of the GP trimer in the structure of GP-benztropine. These two sets of structural changes do not appear to be related to inhibitor binding. The third place where large conformational differences are found is at the $\mathrm{N}$-terminus of the fusion loop (residues 521-525), which is flexible and makes direct interactions with some of the drugs, such as toremifene.

Two Benztropine Molecules Bind within a Single Cavity in EBOV GP. The inhibitor-binding cavity, located between GP1 and GP2, is surrounded by residues from the $\beta 1-\beta 2$ hairpin, $\beta 3, \beta 6$, and $\beta 13$ of GP1, and the stem of the fusion loop $(\beta 19-\beta 20)$ and $\alpha 3$ of GP2. Two benztropine molecules (named $\mathrm{A}$ and $\mathrm{B}$ hereafter) bind in the cavity (Figures 1D and 4A). Benztropine has three rings each connected to a carbon atom acting as a hub (Figure 2). Molecule A has well-defined electron density and binds with one phenyl ring nestling in a subpocket adjacent to $\alpha 3$, delimited by side-chains of residues I 38 and I43 of the $\beta 1-\beta 2$ hairpin, L184 and L186 of $\beta 13$, and L554 and L558 of $\alpha 3$, while the second phenyl ring interacts with V66 of $\beta 3$ and one edge of Y517 from $\beta 19$ (Figures 1 and 4; Figure S3). The center of the molecule is sandwiched by L186 and M548, with all three rings making contact with M548. The second phenyl ring and the azabicyclo ring also make close contacts to both phenyl rings of the $\mathrm{B}$ molecule of benztropine, which has weaker electron density (Figure 1d; Figure S3A). One phenyl ring of benztropine $\mathrm{B}$ makes T-shaped stacking interactions with Y517 and extensive hydrophobic contacts with the side-chain of R64 and A101 on the floor of the binding site. The second phenyl ring points to the solvent and does not interact with any atom 

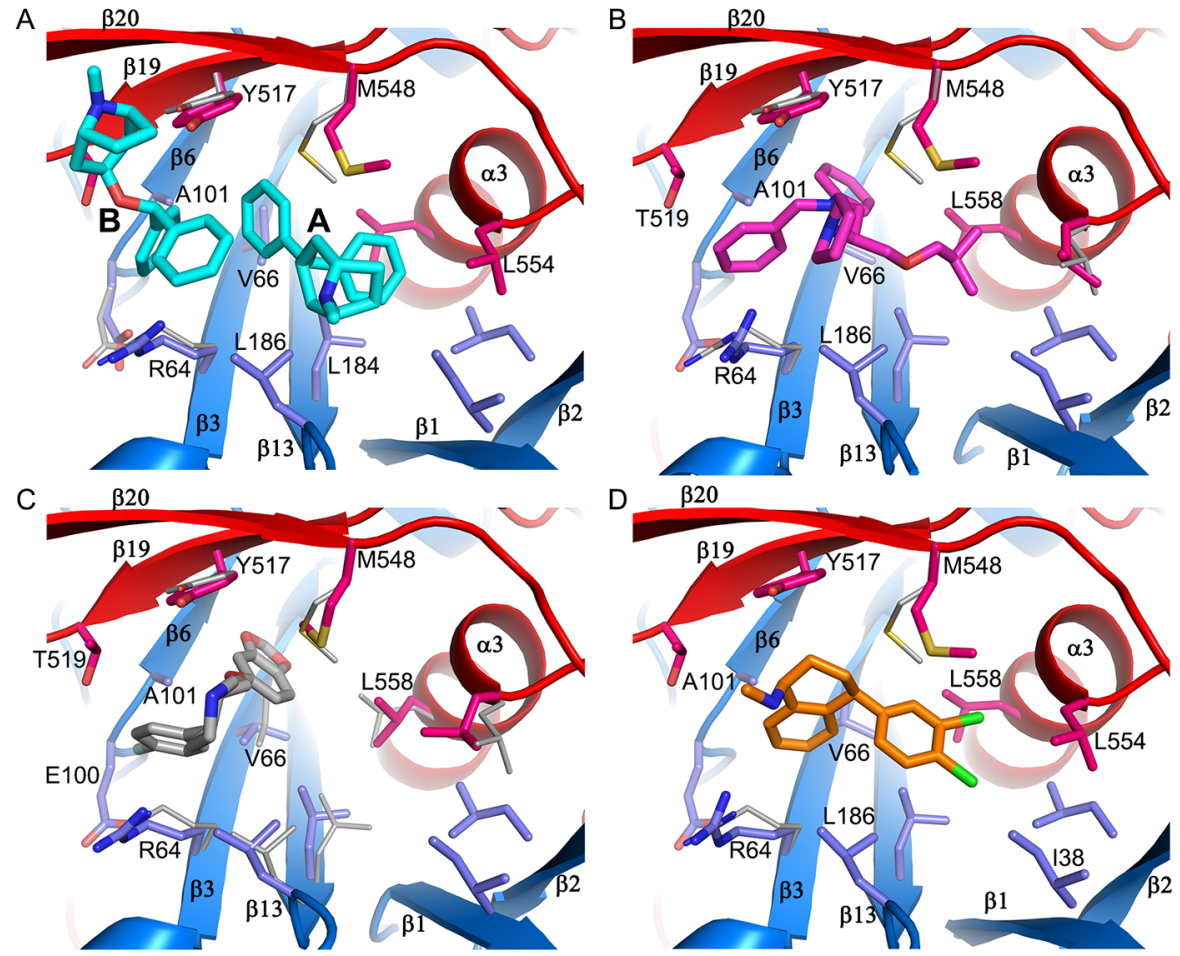

Figure 4. Inhibitor-binding site. (A-D) Details of protein-inhibitor interactions of the GP-benztropine (A), GP-bepridil (B), GP-paroxetine (C), and GP-sertraline (D) complexes. Benztropene, bepridil, paroxetine, and sertraline are shown as cyan, magenta, gray, and orange sticks, respectively. Protein main-chains are shown as ribbons and side-chains as sticks (GP1, blue; GP2, red). Side-chains in the apo GP with significant conformation differences are shown as thinner gray sticks. Residues that interact with the drug $(\leq 3.9 \AA)$ in each panel are labeled. L68 and L515, which also line the binding-site and interact with bepridil and paroxetine (Figure S5), are not shown for clarity.

of the protein. The azabicyclo ring of benztropine $\mathrm{B}$ is not clearly defined in the electron density map (Figure 1D).

Interactions between EBOV GP and Bepridil. Bepridil is located in the binding cavity centered between L186 and Y517. Its phenyl ring binds deep and is fully buried in a hydrophobic subpocket interacting with the side-chains of V66, A101, L515, Y517, and L558. The side-chain of V66 rotates $110^{\circ}$ upon inhibitor binding. The benzyl ring is flanked by R64 and T519 and also interacts with Y517 and A101. The isobutoxy group occupies the subpocket adjacent to $\alpha 3$, interacting with L186, M548, and L558. The pyrrolidine ring is exposed, and only makes contact with the hydroxyl group of Y517 (Figure 4B). Compared to GP-benztropine, bepridil occupies the key volumes in the binding cavity filled by the two benztropine molecules, the ethanamine moiety and the isobutoxy group overlapping with the two phenyl rings of benztropine $A$, while the benzyl and pyrrolidine rings partially overlap with the two phenyl rings of benztropine B (Figure S5).

Paroxetine Occupies Only Part of the Key Binding Volume. Paroxetine binds with its benzodioxol group overlapping the position of the phenyl ring of bepridil and makes interactions with not only the side-chains of V66, A101, L515, Y517, and L558 but also the main-chains of G67 and G102 and side-chain of L68. The side-chain of V66 adopts the conformation seen in the bepridil bound structure, and M548 rotates $140^{\circ}$ around the $\mathrm{C}_{\beta}-\mathrm{C}_{\gamma}$ bond to interact with the yloxymethyl group of paroxetine. The fluorophenyl ring partially overlaps with the benzyl ring of bepridil, but is closer to R64, interacting with the side-chains of R64, T519, A101, and E100. There is an extra blob of electron density in front of M548, which presumably belongs to an unidentified small molecule of either a less-ordered paroxetine or a compound in the sample (purchased from Key Organics with purity >97\%) (Figure 4C; Figure S3B). This volume in front of Y517 and M548 has a propensity to be occupied (including in the previously determined GP-toremifene and GP-ibuprofen structures ${ }^{13}$ ), presumably stabilizing the hydrophobic residues around it. We name this the FF volume since in apo GP it is filled by F193 and F194 of the DFF lid, and its importance for inhibitor binding was suggested from the analysis of the GPtoremifene complex where the chloroethyl group occupies part of the volume in front of M548 and substitution by an ethyl group in tamoxifen or a chlorine in clomiphene greatly reduces binding affinity. ${ }^{13}$ Here, we observe that benztropine A occupies only part of the volume in front of M548 and the second benztropine is required to fill the space in front of Y517, while vice versa, paroxetine binds in part of the volume in front of Y517, with an unidentified small molecule filling the space in front of M548 (Figures S3 and S5).

Interactions between EBOV GP and Sertraline. In contrast to benztropine and paroxetine, sertraline is bound largely in the FF volume. Its dichlorophenyl ring is located at the subpocket adjacent to the $\alpha 3$ helix, interacting with I38, L186, M548, and L558. The position of the dichlorophenyl ring overlaps one of the phenyl rings of benztropine $A$ and the isobutoxy group of bepridil (Figure 4D; Figures S4 and S5). The tetrahydronaphthalene group binds in space between R64 and Y517, making contacts with V66, A101, and R64, and extensive T-shaped ring stacking interactions with Y517. Sertraline is the smallest molecule among the four drugs reported here, but it has better binding affinity and antiviral activity than benztropine (Table 1 ), suggesting that the volume 
A

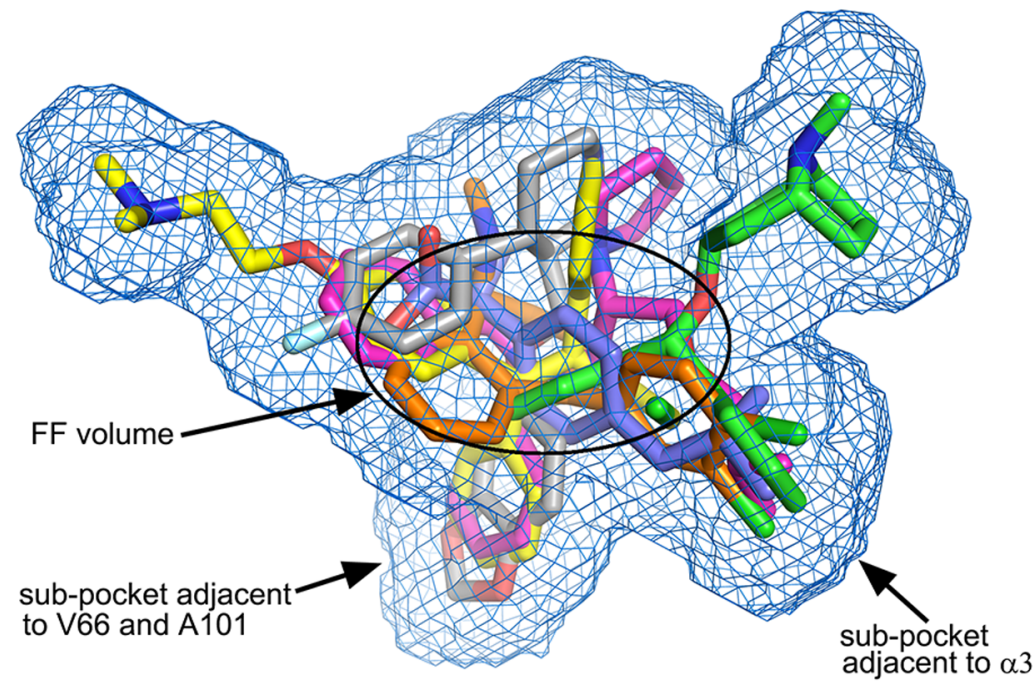

B

\begin{tabular}{|l|l|l|l|l|l|l|}
\hline & toremifene & bepridil & paroxetine & sertraline & ibuprofen & benztropine-A \\
\hline toremifene & 362 & 234 & 198 & 132 & 121 & 86 \\
\hline bepridil & 234 & 332 & 185 & 157 & 134 & 130 \\
\hline paroxetine & 198 & 185 & 277 & 90 & 91 & 42 \\
\hline sertraline & 132 & 157 & 90 & 260 & 142 & 133 \\
\hline ibuprofen & 121 & 134 & 91 & 142 & 188 & 97 \\
\hline benztropine-A & 86 & 130 & 42 & 133 & 97 & 275 \\
\hline
\end{tabular}

Figure 5. Molecular volumes. (A) Volume of EBOV GP drug-binding cavity exploited by the six FDA approved drugs, toremifene (yellow), bepridil (magenta), paroxetine (gray), sertraline (orange), ibuprofen (blue), and benztropine (green). (B) Matrix showing the molecular volumes (gray) of the drugs and common volumes (color coded by size from red to blue) shared by any pair of the drugs. All volumes are in $\AA^{3}$.
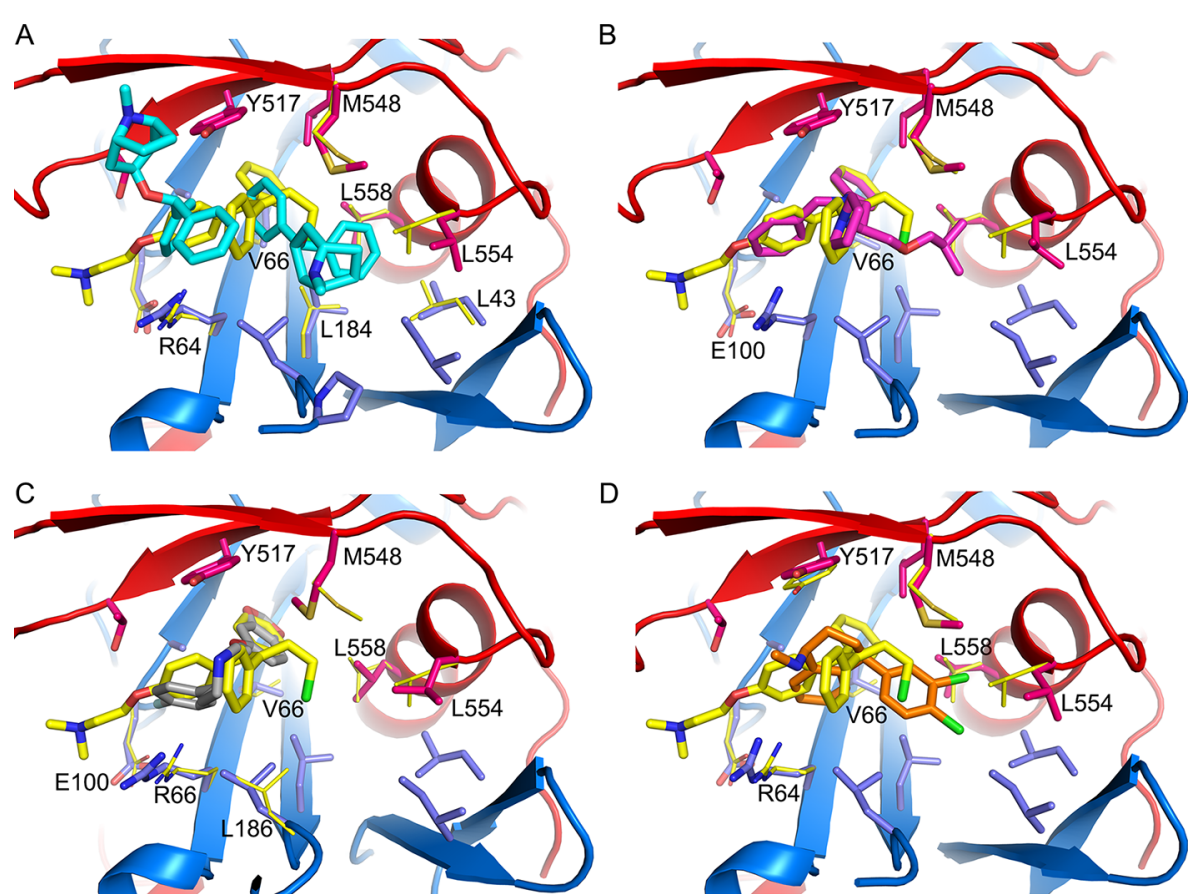

Figure 6. Comparisons with GP-toremifene complex. (A-D) Comparing protein-inhibitor interactions of GP-toremifene with GP-benztropine (A), GP-bepridil (B), GP-paroxetine (C), and GP-sertraline (D). Benztropene, bepridil, paroxetine, and sertraline are shown as cyan, magenta, gray, and orange sticks, respectively, and their associated protein main-chains are shown as ribbons and side-chains as sticks (GP1, blue; GP2, red). Toremifene is drawn as yellow sticks, and side-chains in the GP-toremifene complex with large conformational differences are shown as thinner yellow sticks. 

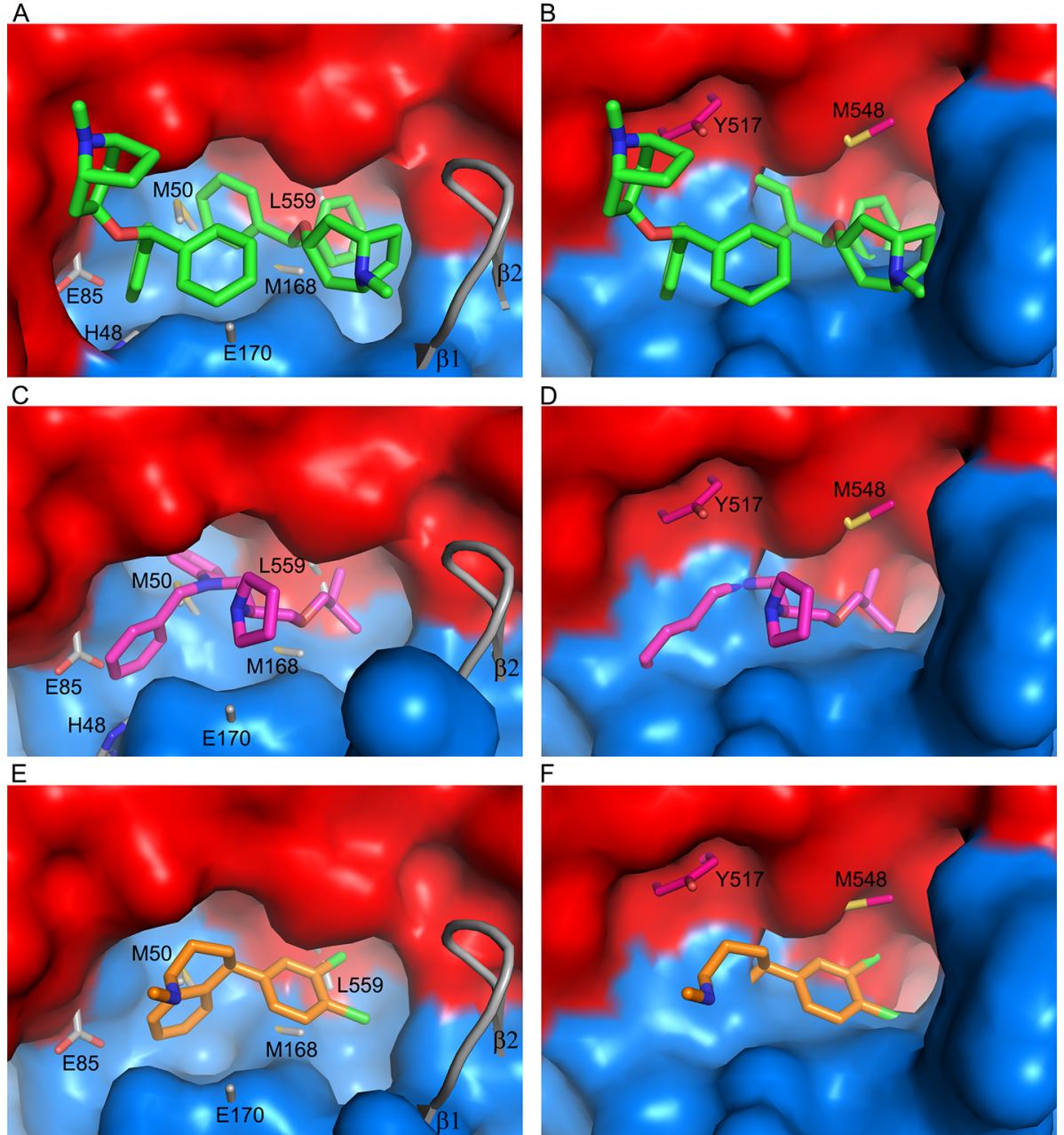

Figure 7. Differences between EBOV and MARV GPs at the inhibitor binding site. Superpositions of the inhibitor binding sites of EBOV GPbenztropine (A,B), GP-bepridil (C,D) and GP-sertraline (E,F) with that of MARV GP. In (A), (C, ) and (E), EBOV GPs are shown as surface representations, inhibitors as sticks, with MARV GP main-chain as gray ribbon and side-chains as gray sticks with labels. In (B), (D), and (F), MARV GP (excluding residues 172-180) is shown as a surface representation, with the EBOV GP complexes shown as ribbons and sticks and side-chains of EBOV GP labeled.

it occupies in the binding cavity and the interactions with the protein are pivotal for inhibitor binding.

None of the Drugs Fully Exploits the Potential Protein-Inhibitor Interactions. The four drugs, despite their chemical diversity, all bind within the same rather extensive cavity of Zaire EBOV GP, which in the case of benztropine accommodates two molecules. Furthermore, the amino acids lining the cavity are highly conserved among the five known species of EBOVs, and so the compounds are likely to interact with GPs of all EBOV species. However, none of the drugs fully exploit the potential protein-inhibitor interactions within the binding pocket. The observed protein-drug interactions are predominantly hydrophobic with no hydrogen bond interactions. There is no significant structural change in the cavity upon drug binding except for some variations in sidechain conformation (Figure S5). Together with the two previously published structures, we have so far determined GP complex structures with six FDA approved drugs. The drug binding cavity has a volume of approximately $1000 \AA^{3}$, while the molecular volumes of the six drugs ranges from the smallest of $188 \AA^{3}$ of ibuprofen to the largest of $362 \AA^{3}$ of toremifene (Figure 5 ). The total volume of the cavity sampled by the six drugs is $712 \AA^{3}$ (excluding the less ordered benztropine B), of which only $25 \AA^{3}$ is common to all. Bepridil and toremifene have the largest common volume of $234 \AA^{3}$, while benztropine A and paroxetine, at $42 \AA^{3}$, share the least. Of the six, toremifene remains the best inhibitor in terms of binding affinity and antiviral activity. Its $K_{\mathrm{d}}$ is 18 -fold better than bepridil and at least 40 -fold better than the other four (Table 1). We compared the four new complexes with GP-toremifene to explore the structure-activity relationship and potential for improvement of the drugs (Figure 6; Figure S6). Toremifene binds with its alkenyl group centered in the FF volume, making extensive interactions to Y517 from its three phenyl rings. This binding mode is most similar to that of bepridil. Ring A of toremifene overlaps with the phenyl ring and ring $\mathrm{C}$ with the benzyl ring of bepridil, while ring B partially overlaps with the pyrrolidine ring of bepridil, but makes more protein contacts. Benztropine and sertraline do not occupy the subpocket where the $\mathrm{A}$ and $\mathrm{B}$ phenyl rings of toremifene bind. The dimethylethanamine of toremifene, to which there is no corresponding group in the other drugs, extends into the main tunnel and is surrounded by polar/charged residues, including R64, E100, T519, T520, and D522. However, there is scope of improvement of toremifene to bind more tightly: (i) substitution of the chlorine by a six-membered ring to explore 
the subpocket adjacent to $\alpha 3$ occupied by a phenyl ring of benztropine A and the isobutoxy group of bepridil; (ii) modification of the dimethylethanamine group to make hydrogen bond interactions with nearby polar/charged residues.

Do These Drugs Bind MARV GP? Toremifene, bepridil, and sertraline have been found to inhibit MARV infection in vitro, ${ }^{15}$ and benztropine was able to block pseudo-MARV entry. ${ }^{21}$ However, MARV GP has only $\sim 27 \%$ sequence identity to EBOV GP (Ravn virus, which causes MARV disease, vs Zaire EBOV virus). The structure of MARV GP in complex with a monoclonal antibody has been published; ${ }^{25}$ however, there are no reports of complexes with putative drugs. Superposition of the structures of MARV and EBOV GP shows that there are significant structural differences at the inhibitor-binding site (Figure 7). The EBOV DFF lid and preceding loop are replaced by a helix in MARV GP, and 15 of the 19 residues that are involved in inhibitor interactions are substituted; in particular, the replacements Ala to Glu at 101 and Val to Met at 66 make the binding cavity much shallower, and the $\beta 1$ $\beta 2$ hairpin of MARV GP folds inward shortening the cavity, while residues Y517 and M548, which make key interactions with inhibitors, both become isoleucine. ${ }^{25}$ Therefore, the shape of the inhibitor-binding cavity in MARV GP is substantially different to that of EBOV GP. Superimpositions of the GPinhibitor complexes onto the structure of MARV GP show the inhibitors make serious steric clashes with MARV GP (Figure 7). If these inhibitors are bound in the same site of MARV, then either their binding modes will differ dramatically or substantial structural changes are required. This observation, together with the fact that two molecules of benztropine bind quite differently in the same pocket, perhaps, illustrate the plasticity of binding when affinity derives simply from hydrophobic interactions without the orientational drivers of charge and hydrogen bond interactions.

\section{CONCLUSIONS}

Structures of two class 1 viral fusion proteins in complexes with small molecule inhibitors have been reported recently, the RSV fusion protein (RSV F) and influenza virus hemagglutinin (HA). ${ }^{26,27}$ Inhibitors of RSV F bind to a hydrophobic pocket adjacent to the fusion peptide around the three-fold symmetry axis with a stoichiometry of one inhibitor per RSV F trimer, ${ }^{26}$ while the inhibitor of flu-HA binds about $16 \AA$ away from the fusion peptide with a stoichiometry of three inhibitors per HA trimer. $^{27}$ For both of these inhibitors, binding stabilizes the fusion proteins, which contrasts with the EBOV GP inhibitors reported here that destabilize the protein by binding directly with the stem of the fusion loop with a stoichiometry of three inhibitors per trimer. Fusion proteins undergo huge structural rearrangement during fusion, supposedly triggered by low $\mathrm{pH}$ and receptor binding in the endo/lysosome, thus viral fusion can be inhibited by either stabilizing or destabilizing the fusion protein by direct binding of small molecule inhibitors. A large number of FDA approved drugs that inhibit EBOV entry have been reported, and various mechanisms of action have been proposed. ${ }^{28-32}$ We have now determined structures of EBOV GP in complexes with six such drugs. These drugs have five different primary pharmacological targets and contain both amphiphiles and cationic amphiphiles; nevertheless, they all bind within the same cavity of EBOV GP. All act to destabilize the GP as shown by thermal shift assay, and this correlates well with their antiviral activity, indicating that these drugs inhibit
EBOV infection via the mechanism of action we proposed previously: inhibitor binding destabilizes GP and triggers premature release of GP2, thereby preventing fusion between the viral and endosome membranes. ${ }^{13}$

\section{EXPERIMENTAL SECTION}

Protein Expression and Purification. The previously described Zaire EBOV (strain Mayinga-76) recombinant glycoprotein extracellular domain construct ${ }^{13}$ with one extra mutation H613A was cloned in the mammalian expression vector pNeosec. ${ }^{33}$ The resulting plasmid pNeosec-GP $\Delta$ has the mucin domain deleted and is tagged with a foldon trimerization sequence from the bacteriophage $\mathrm{T} 4$ fibritin and six histidines at the $\mathrm{C}$ terminus. The endotoxin-free plasmid was transiently transfected into the human embryonic kidney HEK293T (ATCC CRL11268) cells with polyethylenmine (PEI, MW 25kd, Sigma, U.K.). For crystallization, the mannosidase inhibitor kifunensine (Cayman Chemical, Michigan, USA) was added to a final concentration of $5 \mu \mathrm{M}$. The conditioned media was collected 5 days after transfection and dialyzed against PBS. The His-tagged protein was captured with talon beads (Takara Bio Europe SAS, France) at $15{ }^{\circ} \mathrm{C}$ for $1 \mathrm{~h}$ with gentle shaking at $110 \mathrm{rpm}$. The beads were collected and washed in PBS with 5-10 mM imidazole. The protein was eluted with $200 \mathrm{mM}$ imidazole in PBS and further purified by size exclusion chromatography with a Superdex 200 HiLoad 16/600 column (GE healthcare, Buckinghamshire, U.K.) and a buffer of 10 mM MES, pH 5.2, $150 \mathrm{mM} \mathrm{NaCl}$.

Drugs Used in the Experiments. Toremifene (KS-5242), paroxetine hydrochloride hemihydrate (KS-1094), sertraline hydrochloride (KS-1111), aripiprazole (KS-1030), astemizole (KS-5171), efavirenz (KS-5380), and quinacrine dihydrochloride (KH-0002), all with specified purity of $>97 \%$, were purchased from Key Organics. Ibuprofen (Sigma-I4883), benztropine mesylate (Sigma-SML0847), bepridil hydrochloride (Sigma-B5016), strophanthin (AldrichS355445), and U18666A (Sigma-U3633), all with specified purity of $\geq 98 \%$, were purchased from Sigma-Aldrich.

Thermal Shift Assay. Twenty-five microliters of solution containing $2 \mu \mathrm{M}$ glycosylated EBOV GP protein, in a buffer of 25 $\mathrm{mM}$ sodium citrate at $\mathrm{pH} 5.2,150 \mathrm{mM} \mathrm{NaCl}$, and $6 \times$ SYPRO Orange dye (Thermo Fisher Scientific, UK), was mixed with $25 \mu \mathrm{L}$ of compounds in $10 \%$ DMSO containing buffer. Each compound was initially dissolved in 100\% DMSO and then diluted with buffer and DMSO to the desired concentration (except benztropine, which was mixed directly in buffer without DMSO). Then the samples were placed in a semiskirted 96-well PCR plate (4 Titude, Surrey, U.K.), sealed, and heated in an Mx3005p qPCR machine (Stratagene, Agilent Technologies, USA) from room temperature at a rate of $1{ }^{\circ} \mathrm{C} \mathrm{min}{ }^{-1}$ for 74 cycles. Fluorescence changes were monitored with excitation and emission wavelengths at 492 and $610 \mathrm{~nm}$, respectively. Reference wells, i.e., solutions without drugs, but with same amount of DMSO, were used to compare the melting temperature $\left(T_{\mathrm{m}}\right)$. Experiments were carried out in triplicate.

Crystallization and Inhibitor Soaking. The protein was concentrated to $10-12 \mathrm{mg} / \mathrm{mL}$. Crystallization was performed in nanoliter sitting drops (100 nL protein and $100 \mathrm{~nL}$ reservoir) dispensed from a Cartesian robot onto a 96-well Greiner plate. ${ }^{34}$ The crystals were grown in condition containing 9\% (w/v) PEG 6000 and $0.1 \mathrm{M}$ sodium citrate tribasic dihydrate at $\mathrm{pH}$ 5.2. We found microcrystal seeding is an reliable way to obtain reasonable sized crystals in most drops using a previously described method. ${ }^{35}$

GP and inhibitor complexes were obtained by crystal soaking experiments. The crystal soaking solutions were prepared by first dissolving the inhibitors in 100\% DMSO and then diluting the dissolved inhibitors in $15 \%(\mathrm{w} / \mathrm{v})$ PEG 6000 and $0.1 \mathrm{M}$ sodium citrate tribasic dihydrate ( $\mathrm{pH} 5.0$ ) to a final DMSO concentration of $10 \%$ (except for benztropine, which did not require DMSO). The inhibitor concentration was typically from 1 to $10 \mathrm{mM}$ depending on solubility. The crystals were soaked in the above solutions for different lengths of time, ranging from 5 to $20 \mathrm{~min}$. Crystals soaked with inhibitors often resulted in weaker or no diffraction. To obtain the GP-inhibitor 
complexes, we usually had to soak a number of crystals in several concentrations of inhibitor for varying times.

X-ray Data Collection. The inhibitor-soaked crystals were transferred to solutions containing $75 \%$ inhibitor soaking solution and $25 \%(\mathrm{v} / \mathrm{v})$ glycerol for a couple of seconds and then frozen in liquid nitrogen prior to data collection. All data were collected at 100 $\mathrm{K}$ with a frame size of $0.1^{\circ}$ rotation using synchrotron X-rays and PILATUS $6 \mathrm{M}$ detectors at Diamond Light Source, U.K. GPbenztropine and GP-sertraline data were acquired on beamline $\mathrm{I} 02$ with a beam size of $100 \times 20 \mu \mathrm{m}$ and a wavelength of $0.9795 \AA$. The shortest possible exposure time per frame was used. ${ }^{36,37}$ Three hundred and sixty degrees of data of GP-benztropine were collected from a single crystal with an exposure time of $0.04 \mathrm{~s}$ per frame and $40 \%$ beam transmission; $1410^{\circ}$ of data were collected from five crystals with an exposure time of $0.04 \mathrm{~s}$ per frame and $50 \%$ beam transmission for GP-sertraline complex. GP-bepridil and GP-paroxetine data, both using $720^{\circ}$ of data from two crystals, were collected on beamline I24 using an exposure time of $0.01 \mathrm{~s}$ per frame with a beam size of 50 $\times 50 \mu \mathrm{m}^{2}$ and 30 to $50 \%$ beam transmission at wavelength of 0.9686 $\AA$.

Data Processing, Structure Determination and Refinement. Diffraction images were indexed, integrated, and scaled with the automated data processing program Xia2-3dii or Xia2-Dials. ${ }^{38,39}$ The resolution of the diffraction data for these four complexes ranges from 2.07 to $2.40 \AA$ with high redundancy. Each structure was initially phased with rigid-body refinement using the apo GP structure (PDB ID 5JQ3) by omitting residues 190-195 of GP1 and water molecules. The subsequent structure refinement used REFMAC5 $5^{40}$ or PHENIX, ${ }^{41}$ and models were rebuilt with COOT. ${ }^{42}$ All four models were refined to reasonable R-factors with good stereochemistry. Data collection and structure refinement statistics are given in Table S1. Structural comparisons used SHP, ${ }^{43}$ simulated annealing omit electron density maps were calculated with $\mathrm{CNS},{ }^{44}$ volumes of the drug-binding cavity and drug molecules were calculated with VOLUMES (Robert Esnouf, unpublished), and figures were prepared with PyMOL ${ }^{45}$ and LigPlot. $^{46}$

\section{ASSOCIATED CONTENT}

\section{S Supporting Information}

. The Supporting Information is available free of charge on the ACS Publications website at DOI: 10.1021/acs.jmedchem.7b01249.

\section{SMILES (CSV)}

Raw data of thermal-shift assay, structural differences of the four GP-drug complexes, electron density maps for the bound benztropines and paroxetine, LigPlot diagrams of protein inhibitor interactions, comparisons of protein-drug interactions of the four complexes, comparisons of the binding mode of the current four drugs with toremifene, and X-ray data collection and structure refinement statistics (PDF)

\section{Accession Codes}

The coordinates and structure factors have been deposited with the RCSB Protein Data Bank under accession codes 6F6S, $6 \mathrm{~F} 5 \mathrm{U}, 6 \mathrm{~F} 6 \mathrm{I}$, and $6 \mathrm{~F} 6 \mathrm{~N}$ for the GP-benztropine, GP-bepridil, $\mathrm{GP}$-paroxetine, and $\mathrm{GP}$-sertraline, respectively.

\section{AUTHOR INFORMATION}

\section{Corresponding Author}

*Fax: +44 (0)1865 287501. E-mail: dave@strubi.ox.ac.uk.

\section{ORCID}

David I. Stuart: 0000-0002-3426-4210

\section{Author Contributions}

${ }^{\#}$ These authors contributed equally to this work. J.R., Y.Z., and D.I.S. designed the project. J.R. and Y.Z. performed experi- ments. J.R., Y.Z., and D.I.S. analyzed the results together with E.E.F. and wrote the manuscript. All authors read and approved the manuscript.

\section{Notes}

The authors declare no competing financial interest.

\section{ACKNOWLEDGMENTS}

The authors would like to thank Diamond Light Source for beamtime (proposal mx10627) and the staff of beamlines I02 and I24 for assistance with crystal testing and data collection. Y.Z. was supported by the Biostruct-X project (283570) funded by the EU seventh Framework Programme (FP7), J.R. by the Wellcome Trust, and D.I.S. by the UK Medical Research Council (MR/N00065X/1). This is a contribution from the UK Instruct Centre. The Wellcome Trust Centre for Human Genetics is supported by the Wellcome Trust (grant 090532/ $\mathrm{Z} / 09 / \mathrm{Z}$ )

\section{ABBREVIATIONS USED}

DMSO, dimethyl sulfoxide; EBOV, Ebolavirus; flu-HA, influenza virus hemagglutinin; FDA, Food and Drug Administration; GP, glycoprotein; MARV, Marburgvirus; PEI, polyethylenmine; RSV, respiratory syncytial virus; RSV F, respiratory syncytial virus fusion protein.

\section{REFERENCES}

(1) Kuhn, J. H.; Becker, S.; Ebihara, H.; Geisbert, T. W.; Johnson, K. M.; Kawaoka, Y.; Lipkin, W. I.; Negredo, A. I.; Netesov, S. V.; Nichol, S. T.; Palacios, G.; Peters, C. J.; Tenorio, A.; Volchkov, V. E.; Jahrling, P. B. Proposal for a revised taxonomy of the family Filoviridae: classification, names of taxa and viruses, and virus abbreviations. Arch. Virol. 2010, 155, 2083-2103.

(2) Anthony, S. M.; Bradfute, S. B. Filoviruses: One of these things is (not) like the other. Viruses 2015, 7, 5172-5190.

(3) Coltart, C. E.; Lindsey, B.; Ghinai, I.; Johnson, A. M.; Heymann, D. L. The Ebola outbreak, 2013-2016: old lessons for new epidemics. Philos. Trans. R. Soc., B 2017, 372, 1-24.

(4) Carette, J. E.; Raaben, M.; Wong, A. C.; Herbert, A. S.; Obernosterer, G.; Mulherkar, N.; Kuehne, A. I.; Kranzusch, P. J.; Griffin, A. M.; Ruthel, G.; Dal Cin, P.; Dye, J. M.; Whelan, S. P.; Chandran, K.; Brummelkamp, T. R. Ebola virus entry requires the cholesterol transporter Niemann-Pick C1. Nature 2011, 477, 340343.

(5) Hacke, M.; Bjorkholm, P.; Hellwig, A.; Himmels, P.; de Almodovar, C. R.; Brugger, B.; Wieland, F.; Ernst, A. M. Inhibition of Ebola virus glycoprotein-mediated cytotoxicity by targeting its transmembrane domain and cholesterol. Nat. Commun. 2015, 6, 7688 .

(6) Nanbo, A.; Imai, M.; Watanabe, S.; Noda, T.; Takahashi, K.; Neumann, G.; Halfmann, P.; Kawaoka, Y. Ebolavirus is internalized into host cells via macropinocytosis in a viral glycoprotein-dependent manner. PLoS Pathog. 2010, 6, e1001121.

(7) Saeed, M. F.; Kolokoltsov, A. A.; Albrecht, T.; Davey, R. A. Cellular entry of ebola virus involves uptake by a macropinocytosis-like mechanism and subsequent trafficking through early and late endosomes. PLoS Pathog. 2010, 6, e1001110.

(8) Takada, A.; Robison, C.; Goto, H.; Sanchez, A.; Murti, K. G.; Whitt, M. A.; Kawaoka, Y. A system for functional analysis of Ebola virus glycoprotein. Proc. Natl. Acad. Sci. U. S. A. 1997, 94, 1476414769 .

(9) Lee, J. E.; Fusco, M. L.; Hessell, A. J.; Oswald, W. B.; Burton, D. R; Saphire, E. O. Structure of the Ebola virus glycoprotein bound to an antibody from a human survivor. Nature 2008, 454, 177-182.

(10) Wang, H.; Shi, Y.; Song, J.; Qi, J.; Lu, G.; Yan, J.; Gao, G. F. Ebola viral glycoprotein bound to its endosomal receptor NiemannPick C1. Cell 2016, 164, 258-268. 
(11) Zhao, Y.; Ren, J.; Harlos, K.; Stuart, D. I. Structure of glycosylated NPC1 luminal domain C reveals insights into NPC2 and Ebola virus interactions. FEBS Lett. 2016, 590, 605-612.

(12) Weissenhorn, W.; Carfi, A.; Lee, K. H.; Skehel, J. J.; Wiley, D. C. Crystal structure of the Ebola virus membrane fusion subunit, GP2, from the envelope glycoprotein ectodomain. Mol. Cell 1998, 2, 605616.

(13) Zhao, Y.; Ren, J.; Harlos, K.; Jones, D. M.; Zeltina, A.; Bowden, T. A.; Padilla-Parra, S.; Fry, E. E.; Stuart, D. I. Toremifene interacts with and destabilizes the Ebola virus glycoprotein. Nature 2016, 535, 169-172.

(14) Johansen, L. M.; Brannan, J. M.; Delos, S. E.; Shoemaker, C. J.; Stossel, A.; Lear, C.; Hoffstrom, B. G.; Dewald, L. E.; Schornberg, K. L.; Scully, C.; Lehar, J.; Hensley, L. E.; White, J. M.; Olinger, G. G. FDA-approved selective estrogen receptor modulators inhibit Ebola virus infection. Sci. Transl. Med. 2013, 5, 190 ra79.

(15) Johansen, L. M.; DeWald, L. E.; Shoemaker, C. J.; Hoffstrom, B. G.; Lear-Rooney, C. M.; Stossel, A.; Nelson, E.; Delos, S. E.; Simmons, J. A.; Grenier, J. M.; Pierce, L. T.; Pajouhesh, H.; Lehar, J.; Hensley, L. E.; Glass, P. J.; White, J. M.; Olinger, G. G. A screen of approved drugs and molecular probes identifies therapeutics with anti-Ebola virus activity. Sci. Transl. Med. 2015, 7, 290 ra89.

(16) Kouznetsova, J.; Sun, W.; Martinez-Romero, C.; Tawa, G.; Shinn, P.; Chen, C. Z.; Schimmer, A.; Sanderson, P.; McKew, J. C.; Zheng, W.; Garcia-Sastre, A. Identification of 53 compounds that block Ebola virus-like particle entry via a repurposing screen of approved drugs. Emerging Microbes Infect. 2014, 3, e84.

(17) Yermolina, M. V.; Wang, J.; Caffrey, M.; Rong, L. L.; Wardrop, D. J. Discovery, synthesis, and biological evaluation of a novel group of selective inhibitors of filoviral entry. J. Med. Chem. 2011, 54, 765-781.

(18) Edwards, M. R.; Pietzsch, C.; Vausselin, T.; Shaw, M. L.; Bukreyev, A.; Basler, C. F. High-throughput minigenome system for identifying small-molecule inhibitors of Ebola virus replication. ACS Infect. Dis. 2015, 1, 380-387.

(19) Anantpadma, M.; Kouznetsova, J.; Wang, H.; Huang, R.; Kolokoltsov, A.; Guha, R.; Lindstrom, A. R.; Shtanko, O.; Simeonov, A.; Maloney, D. J.; Maury, W.; LaCount, D. J.; Jadhav, A.; Davey, R. A. Large-scale screening and identification of novel Ebola virus and Marburg virus entry inhibitors. Antimicrob. Agents Chemother. 2016, 60, $4471-4481$.

(20) Basu, A.; Li, B.; Mills, D. M.; Panchal, R. G.; Cardinale, S. C.; Butler, M. M.; Peet, N. P.; Majgier-Baranowska, H.; Williams, J. D.; Patel, I.; Moir, D. T.; Bavari, S.; Ray, R.; Farzan, M. R.; Rong, L.; Bowlin, T. L. Identification of a small-molecule entry inhibitor for filoviruses. J. Virol. 2011, 85, 3106-3119.

(21) Cheng, H.; Lear-Rooney, C. M.; Johansen, L.; Varhegyi, E.; Chen, Z. W.; Olinger, G. G.; Rong, L. Inhibition of Ebola and Marburg virus entry by $\mathrm{G}$ protein-coupled receptor antagonists. J. Virol. 2015, 89, 9932-9938.

(22) Madrid, P. B.; Panchal, R. G.; Warren, T. K.; Shurtleff, A. C.; Endsley, A. N.; Green, C. E.; Kolokoltsov, A.; Davey, R.; Manger, I. D.; Gilfillan, L.; Bavari, S.; Tanga, M. J. Evaluation of Ebola virus inhibitors for drug repurposing. ACS Infect. Dis. 2015, 1, 317-326.

(23) Madrid, P. B.; Chopra, S.; Manger, I. D.; Gilfillan, L.; Keepers, T. R.; Shurtleff, A. C.; Green, C. E.; Iyer, L. V.; Dilks, H. H.; Davey, R. A.; Kolokoltsov, A. A.; Carrion, R., Jr.; Patterson, J. L.; Bavari, S.; Panchal, R. G.; Warren, T. K.; Wells, J. B.; Moos, W. H.; Burke, R. L.; Tanga, M. J. A systematic screen of FDA-approved drugs for inhibitors of biological threat agents. PLoS One 2013, 8, e60579.

(24) Lu, F.; Liang, Q.; Abi-Mosleh, L.; Das, A.; De Brabander, J. K.; Goldstein, J. L.; Brown, M. S. Identification of NPC1 as the target of U18666A, an inhibitor of lysosomal cholesterol export and Ebola infection. eLife 2015, 4, 1-16.

(25) Hashiguchi, T.; Fusco, M. L.; Bornholdt, Z. A.; Lee, J. E.; Flyak, A. I.; Matsuoka, R.; Kohda, D.; Yanagi, Y.; Hammel, M.; Crowe, J. E., Jr.; Saphire, E. O. Structural basis for Marburg virus neutralization by a cross-reactive human antibody. Cell 2015, 160, 904-912.

(26) Battles, M. B.; Langedijk, J. P.; Furmanova-Hollenstein, P.; Chaiwatpongsakorn, S.; Costello, H. M.; Kwanten, L.; Vranckx, L.;
Vink, P.; Jaensch, S.; Jonckers, T. H.; Koul, A.; Arnoult, E.; Peeples, M. E.; Roymans, D.; McLellan, J. S. Molecular mechanism of respiratory syncytial virus fusion inhibitors. Nat. Chem. Biol. 2016, 12, 87-93.

(27) Kadam, R. U.; Wilson, I. A. Structural basis of influenza virus fusion inhibition by the antiviral drug Arbidol. Proc. Natl. Acad. Sci. U. S. A. 2017, 114, 206-214.

(28) Miller, M. E.; Adhikary, S.; Kolokoltsov, A. A.; Davey, R. A. Ebolavirus requires acid sphingomyelinase activity and plasma membrane sphingomyelin for infection. J. Virol. 2012, 86, 7473-7483.

(29) Gehring, G.; Rohrmann, K.; Atenchong, N.; Mittler, E.; Becker, S.; Dahlmann, F.; Pohlmann, S.; Vondran, F. W.; David, S.; Manns, M. P.; Ciesek, S.; von Hahn, T. The clinically approved drugs amiodarone, dronedarone and verapamil inhibit filovirus cell entry. J. Antimicrob. Chemother. 2014, 69, 2123-2131.

(30) Fan, H.; Du, X.; Zhang, J.; Zheng, H.; Lu, X.; Wu, Q.; Li, H.; Wang, H.; Shi, Y.; Gao, G.; Zhou, Z.; Tan, D. X.; Li, X. Selective inhibition of Ebola entry with selective estrogen receptor modulators by disrupting the endolysosomal calcium. Sci. Rep. 2017, 7, 41226.

(31) Shoemaker, C. J.; Schornberg, K. L.; Delos, S. E.; Scully, C.; Pajouhesh, H.; Olinger, G. G.; Johansen, L. M.; White, J. M. Multiple cationic amphiphiles induce a Niemann-Pick $C$ phenotype and inhibit Ebola virus entry and infection. PLoS One 2013, 8, e56265.

(32) Sakurai, Y.; Kolokoltsov, A. A.; Chen, C. C.; Tidwell, M. W.; Bauta, W. E.; Klugbauer, N.; Grimm, C.; Wahl-Schott, C.; Biel, M.; Davey, R. A. Ebola virus. Two-pore channels control Ebola virus host cell entry and are drug targets for disease treatment. Science 2015, 347, 995-998.

(33) Zhao, Y.; Ren, J.; Padilla-Parra, S.; Fry, E. E.; Stuart, D. I. Lysosome sorting of beta-glucocerebrosidase by LIMP-2 is targeted by the mannose 6-phosphate receptor. Nat. Commun. 2014, 5, 4321.

(34) Walter, T. S.; Diprose, J. M.; Mayo, C. J.; Siebold, C.; Pickford, M. G.; Carter, L.; Sutton, G. C.; Berrow, N. S.; Brown, J.; Berry, I. M.; Stewart-Jones, G. B.; Grimes, J. M.; Stammers, D. K.; Esnouf, R. M.; Jones, E. Y.; Owens, R. J.; Stuart, D. I.; Harlos, K. A procedure for setting up high-throughput nanolitre crystallization experiments. Crystallization workflow for initial screening, automated storage, imaging and optimization. Acta Crystallogr., Sect. D: Biol. Crystallogr. 2005, 61, 651-657.

(35) Walter, T. S.; Mancini, E. J.; Kadlec, J.; Graham, S. C.; Assenberg, R.; Ren, J.; Sainsbury, S.; Owens, R. J.; Stuart, D. I.; Grimes, J. M.; Harlos, K. Semi-automated microseeding of nanolitre crystallization experiments. Acta Crystallogr., Sect. F: Struct. Biol. Cryst. Commun. 2008, 64, 14-18.

(36) Owen, R. L.; Axford, D.; Nettleship, J. E.; Owens, R. J.; Robinson, J. I.; Morgan, A. W.; Dore, A. S.; Lebon, G.; Tate, C. G.; Fry, E. E.; Ren, J.; Stuart, D. I.; Evans, G. Outrunning free radicals in room-temperature macromolecular crystallography. Acta Crystallogr., Sect. D: Biol. Crystallogr. 2012, 68, 810-818.

(37) Owen, R. L.; Paterson, N.; Axford, D.; Aishima, J.; SchulzeBriese, C.; Ren, J.; Fry, E. E.; Stuart, D. I.; Evans, G. Exploiting fast detectors to enter a new dimension in room-temperature crystallography. Acta Crystallogr., Sect. D: Biol. Crystallogr. 2014, 70, 1248-1256.

(38) Winter, G.; Lobley, C. M.; Prince, S. M. Decision making in xia2. Acta Crystallogr., Sect. D: Biol. Crystallogr. 2013, 69, 1260-1273.

(39) Waterman, D. G.; Winter, G.; Gildea, R. J.; Parkhurst, J. M.; Brewster, A. S.; Sauter, N. K.; Evans, G. Diffraction-geometry refinement in the DIALS framework. Acta Crystallogr. D Struct. Biol. 2016, 72, 558-575.

(40) Murshudov, G. N.; Skubak, P.; Lebedev, A. A.; Pannu, N. S.; Steiner, R. A.; Nicholls, R. A.; Winn, M. D.; Long, F.; Vagin, A. A. REFMAC5 for the refinement of macromolecular crystal structures. Acta Crystallogr., Sect. D: Biol. Crystallogr. 2011, 67, 355-367.

(41) Janowski, P. A.; Moriarty, N. W.; Kelley, B. P.; Case, D. A.; York, D. M.; Adams, P. D.; Warren, G. L. Improved ligand geometries in crystallographic refinement using AFITT in PHENIX. Acta Crystallogr. D Struct. Biol. 2016, 72, 1062-1072.

(42) Emsley, P.; Cowtan, K. Coot: model-building tools for molecular graphics. Acta Crystallogr., Sect. D: Biol. Crystallogr. 2004, $60,2126-2132$. 
(43) Stuart, D. I.; Levine, M.; Muirhead, H.; Stammers, D. K. Crystal structure of cat muscle pyruvate kinase at a resolution of $2.6 \mathrm{~A}$. J. Mol. Biol. 1979, 134, 109-142.

(44) Brunger, A. T. Version 1.2 of the Crystallography and NMR system. Nat. Protoc. 2007, 2, 2728-2733.

(45) DeLano, W. L.; Lam, J. W. PyMOL: A communications tool for computational models. Abstracts of Papers of the American Chemical Society 2005, 230, U1371-U1372.

(46) Laskowski, R. A.; Swindells, M. B. LigPlot+: multiple ligandprotein interaction diagrams for drug discovery. J. Chem. Inf. Model. 2011, 51, 2778-2786. 